\title{
Development and behavior of Plaintive Cuckoo (Cacomantis merulinus) nestlings and their Common Tailorbird (Orthotomus sutorius) hosts
}

Odd Helge Tunheim ${ }^{1}$, Bård G. Stokke ${ }^{1,2}$, Longwu Wang ${ }^{3}$, Canchao Yang ${ }^{4}$, Aiwu Jiang ${ }^{5}$, Wei Liang ${ }^{4}$, Eivin Røskaft ${ }^{1}$ and Frode Foss $\varnothing \mathrm{y}^{1,2^{*}}$ (D)

\begin{abstract}
Background: Our knowledge of avian brood parasitism is primarily based on studies of a few selected species. Recently, researchers have targeted a wider range of host-parasite systems, which has allowed further evaluation of hypotheses derived from well-known study systems but also disclosed adaptations that were previously unknown. Here we present developmental and behavioral data on the previously undescribed Plaintive Cuckoo (Cacomantis merulinus) nestling and one of its hosts, the Common Tailorbird (Orthotomus sutorius).
\end{abstract}

Methods: We discovered more than 80 Common Tailorbird nests within an area of $25 \mathrm{~km}^{2}$, and we recorded nestling characteristics, body mass, tarsus length and begging display every 3 days for both species.

Results: Plaintive Cuckoo nestlings followed a developmental pathway that was relatively similar to that of their well-studied relative, the Common Cuckoo (Cuculus canorus). Tailorbird foster siblings were evicted from the nest rim. The cuckoo nestlings gained weight faster than host nestlings, and required 3-9 days longer time to fledge than host nestlings. Predation was high during the early stages of development, but the nestlings acquired a warning display around 11 days in the nest, after which none of the studied cuckoo nestlings were depredated. The cuckoos' begging display, which appeared more intense than that of host nestlings, was initially vocally similar with that of the host nestlings but began to diverge from the host sound output after day 9 .

Conclusions: The developmental data on Plaintive Cuckoo nestlings and their tailorbird host builds an important foundation for future work on the co-evolutionary interactions in this parasite-host system.

Keywords: Avian brood parasitism, Coevolution, Cuckoos, Developmental biology

\section{Background}

The cuckoo family, Cuculidae, is a phylogenetic group of birds renowned for its large number of brood parasites (40\% of cuckoos). The parasitic reproductive strategy has likely evolved independently three times in the lineage (Payne 2005; Sorenson and Payne 2005). Many cuckoo species are difficult to study, as they live in the

\footnotetext{
*Correspondence: frode.fossoy@ntnu.no

${ }^{1}$ Department of Biology, Norwegian University of Science

and Technology (NTNU), 7491 Trondheim, Norway

Full list of author information is available at the end of the article
}

tropics where the birds are difficult to observe and the nests are well hidden. Hence, the majority of our knowledge of brood parasitism has been acquired from investigations on a few species [particularly the Common Cuckoo (Cuculus canorus)] (Payne 2005; Stevens 2013). Recently, the focus has broadened to a wider range of host-parasite systems, which has strengthened some of the hypotheses derived from early studies and countered others (Stevens 2013). In addition, different hostparasite systems are at different stages as they respond to various ecological selection pressures and hence follow 
various co-evolutionary routes (Britton et al. 2007; Stevens 2013). Species in east and southeast Asia and New Guinea are especially poorly studied, so explorations of these systems may reveal novel deceptive traits (Stevens 2013). The present study aims to shed light on a poorly studied host-parasite system in southwestern China that includes the Plaintive Cuckoo (Cacomantis merulinus) (hereafter referred to as "cuckoo"), a small Asian cuckoo, and one of its hosts, the Common Tailorbird (Orthotomus sutorius) (hereafter referred to as "tailorbird").

Many parasite nestlings eliminate host progeny, an adaptation that is energetically costly but that results in a monopoly of parental provisioning by the brood parasitic nestling (Grim et al. 2009). For many cuckoo species, the elimination occurs by the cuckoo nestling evicting host eggs and/or nestlings off the nest rim to certain death (Davies 2000).

Non-evicting brood parasites grow up alongside host nestlings and have to compete with them for parental resources (Kilner et al. 2004). The strategy of these nestlings is to become stronger competitors that the parents will favor over their own young (Soler et al. 1995). As parents adjust their investment according to the number of nestlings in the nest, the presence of foster siblings may reward parasites by positively affecting the number of food loads that host parents bring to the nest (Kilner et al. 2004).

By contrast, evictors face problems in assimilating sufficient food, as host parents adjust their provisioning in response to the gape-area displayed in the nest. Even though cuckoo nestlings have a relatively large, conspicuous gape, it is far smaller than the area that would be covered by an entire brood (Kilner and Davies 1999). Therefore, the cuckoo nestling employs another set of adaptations to ensure that it will receive sufficient provisioning: a set of exaggerated begging signals. A single Common Cuckoo nestling can mimic the sound output of a whole brood of Reed Warblers (Acrocephalus scirpaceus) by day 6 or 7 after hatching, producing a begging call that increases with age in frequency (Davies et al. 1998; Kilner et al. 1999). Thereby, evictors are able to elicit sufficient amounts of food, as they normally are of larger size than the selected hosts. In some broodparasitic systems, mimicry of the host nestling begging call may also be a trait acquired to battle rejection by host parents (Langmore et al. 2003) or to convince host parents of nestling value to avoid desertion, as host parents may desert progeny that take an atypical long time to fledge (Grim et al. 2003; Grim 2007). Other traits have also been revealed to act as solicitation enhancers, such as the colorful gapes of the Common Cuckoo nestling that work as a supernormal stimulus for Rufous Bush Chats (Erythropygia galactotes) (Álvarez 2004), but not for Dunnocks (Prunella modularis), Robins (Erithacus rubecula) and Reed Warblers (Noble et al. 1999) and gape-colored skin patches on the wings of Hodgson's Hawk-cuckoo (Cuculus fugax) nestlings that simulate additional gapes in the nest (Tanaka and Ueda 2005).

Here, we describe nestling development of the Plaintive Cuckoo and its Common Tailorbird host in southwestern China. Previous studies of this system examined adaptations at the egg stage; the host has evolved dimorphic eggs that are mimicked by the cuckoo, and mismatched cuckoo eggs are frequently rejected (Yang et al. 2016; Liang et al. 2017). Both species have two egg morphs, featuring a ground color of either blue or white and spots that are brown or red. Within each tailorbird clutch, only one egg type is found. A recent study reported that the Plaintive Cuckoo does not always deposit eggs that match the host egg morph. The result is a $50 \%$ mismatch of the host egg morph by the cuckoo, and such mismatches do lead to host rejection of the cuckoo egg (Yang et al. 2016). The co-evolutionary interactions at the nestling stage in this host-parasite system are, however, unknown (Payne 2005). The aim of this paper is therefore to describe the behavior and collect developmental data of the Plaintive Cuckoo nestling. Such knowledge will have fundamental importance for further studies of this system and will enable comparisons with other host-parasite systems.

\section{Methods}

\section{Study area and study species}

This study was conducted in and around the village of Nonggang $\left(22^{\circ} 30^{\prime} \mathrm{N}, 106^{\circ} 58^{\prime} \mathrm{E}\right)$, Guangxi Zhuang Autonomous Region, in southwestern China. The landscape is a mosaic of steep limestone peaks covered in dense vegetation amid cultivated flatlands. The area is situated on the margins of the tropics with a mean annual rainfall of 1150-1550 $\mathrm{mm}$ and a mean annual temperature of 20.8$22.4{ }^{\circ} \mathrm{C}$ (Yang et al. 2016). The fieldwork was performed from April to June 2014.

The Common Tailorbird, an abundant species in the inhabited parts of the Nonggang area, is a common host for the Plaintive Cuckoo (Payne 2005; Yang et al. 2016). It uses sturdy leaves from a variety of tree species to create its nest, sewing one or two leaves together with organic material (Fig. 1). The result is a dome-shaped fundament in which the tailorbird places its nest. Nonggang is on the northern boundary of the Plaintive Cuckoo's distribution range and this species migrates from its breeding areas in China to spend the winter in the more southern parts of its natural range (Payne 2005; Yang et al. 2012). Although some previous studies of the Plaintive Cuckoo have been conducted, the co-evolutionary interactions between the cuckoo and its tailorbird host have remained largely unknown. The Plaintive Cuckoo is a relatively 
small cuckoo where females either look similar to males or appear in a reddish-brown "hepatic" morph (Payne 2005). In the Nonggang area, $15-18 \%$ of tailorbird nests were parasitized (Yang et al. 2016).

\section{Data collection}

Nests were found by systematically searching habitats suitable for nesting tailorbirds, whereby the nest locations were plotted on a GPS (Oregon 450, Garmin International Inc., Kansas, USA) and nest details (egg morph, number of eggs, etc.) were noted. When a new nest with eggs was found, a floating test was used to determine the developmental stage (for details, see Ackerman and Eagles-Smith 2010), and nests with remaining tailorbird eggs were checked daily from when the expected hatching day approached and afterwards. To reduce predation and host rejection of mismatched eggs, cuckoo eggs were moved to an incubator until they hatched (Brinsea Mini EX, Brinsea Products Ltd, Weston Super Mare, UK), where they were maintained at $37.5{ }^{\circ} \mathrm{C}$ and a humidity level of $45 \%$. Although it would have been preferable to return each nestling to its natal nest, high predation of the nests and unsuitable nest locations (in two situations) made this impossible and only a minority of the nestlings were reintroduced to their natal nests. Newly hatched cuckoos were translocated to nests at different brooding stages, depending on nest availability.

For comparing development and behavior of parasite and host nestlings, we experimentally manipulated hosts nests to contain a single host nestling only. The single tailorbird nestlings were cross-fostered, with the exception of two individuals that were kept in their natal nests. Two cuckoo nestlings were placed in their original nests,

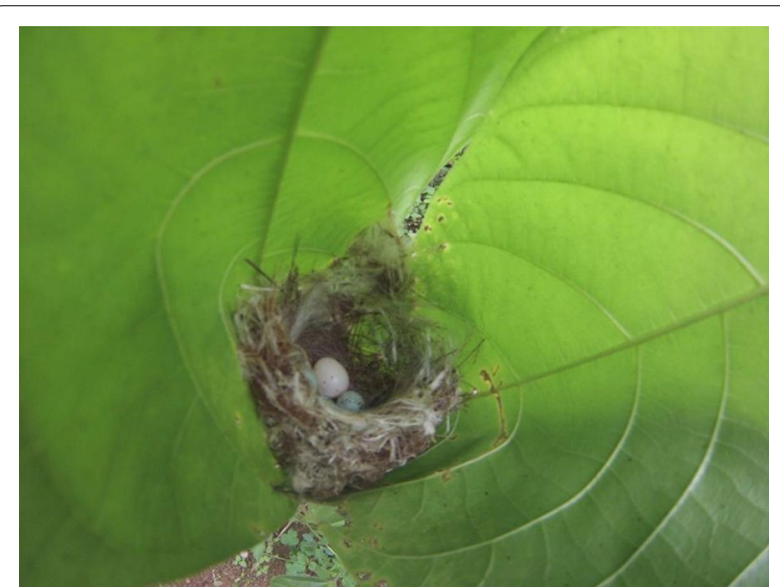

Fig. 1 A typical tailorbird nest neatly placed on a foundation created by sewing one or two leaves together. The tailorbird egg clutch is the blue morph and has been parasitized by a Plaintive Cuckoo of the white egg morph whereas the remaining eleven cuckoo nestlings were translocated to new nests. One cuckoo nestling hatched on the day of departure, and was thus disregarded from the experimental nest count, but measurements taken after hatching was used. The brooding stage of the manipulated tailorbird nests varied and eggs that were already present were removed on day 2 or 3 in the onehost group to provide the parents with a gradual transition between brooding and hatching. Host eggs were not removed from the nests unless the cuckoo nestling failed to evict them. In two cases, cuckoo nestlings hatched in tailorbird nests that were in inaccessible locations. After weight measurements and developmental traits were recorded, the nestlings were translocated to new nests. Nests that were found unexpectedly empty were assumed to have experienced predation. However, environmental factors such as heavy rainfall that caused flooding or nest destruction might have caused the nest content to vanish.

Data was acquired from both Plaintive Cuckoo nestlings and tailorbird nestlings every 3 days after hatching (day 1) in order to compare their development in tailorbird nests. Given the temporal difference in post-natal development between the cuckoo and tailorbird, cuckoo nestlings were followed from day 1 to day 18 whereas the tailorbird nestlings were followed from day 1 to day 12 . The majority of cuckoo nestlings were monitored more frequently than the 3-day intervals in order to document their development, but the sampling was not methodologically structured. The observation of nestling development followed the procedures of Jonsomjit et al. (2007). We did not follow up the nestlings after fledging, and post-fledging care was therefore not observed.

Nest activity was recorded with a sound recorder, a Zoom H4N (Zoom Corporation, Tokyo, Japan), and four pen cameras, Hyundai model HYM-V808 (Hyundai Motor Group, Seoul, South Korea), on specified days. The recorders were attached to a portable bamboo stick or to natural vegetation around the nest and were concealed with leaves to make them as cryptic as possible. The recorder placement was standardized as much as possible, with the pen camera ideally capturing the nest entrance and a portion of the parental activity around the nest. Average length of video recordings was $91.4 \mathrm{~min}$ for nests containing one tailorbird nestling and $102.6 \mathrm{~min}$ for nests containing one cuckoo nestling. A total of 47 video recordings of cuckoo nestlings and 27 recordings of one tailorbird nestlings were completed, spread out over various ages. Sound recordings were performed in the field (cuckoos: $n=28$, tailorbirds: $n=9$ ) for cuckoo and host nestlings on days 3 and 6. The duration of field recordings was approximately $2 \mathrm{~h}$. The sound recorder was optimally placed between 10 and $15 \mathrm{~cm}$ from the nest entrance with both microphones directed toward the nest opening. Recordings that were 
too noisy or did not capture begging calls were ignored in the analysis. A digitizer incorporated in the sound recorder sampled the recordings at $44.1 \mathrm{kHz}$ with 16 bits per sample. Analysis of the sound recordings was performed using RavenPro 1.5 (Bioacoustics Research Program 2014). The settings used for the spectrogram analysis were a 256-sample Hann window, 3-dB filter bandwidth of $248 \mathrm{~Hz}$, time grid resolution of 128 sample with $50 \%$ overlap, and a discrete Fourier transformation size of 256 samples.

\section{Growth measurements}

Measurements of the tarsus and body weight were taken using a vernier caliper and an electronic balance. All measurements were taken by one person (O.H.T.) following standard methods. The electronic balance was calibrated before each weighing and the weight was recorded to the nearest $0.01 \mathrm{~g}$. The tarsus length was measured with one electronic caliper to the nearest $0.01 \mathrm{~mm}$, by which the technique of bending the toes back to a $90^{\circ}$ angle was used (see Svensson 1992). Thus, the tarsus was measured between the back of the intertarsal joint to the bending point between the toes and the tarsus. All statistical calculations were executed in R (R Core Team 2013), graphs were made using the "sciplot" package (Morales and Murdoch 2012) and standard linear models were used to test for significance levels.

\section{Results}

\section{General results}

More than 80 Common Tailorbird nests were found within an area of $25 \mathrm{~km}^{2}$. However, due to high predation rate $(\sim 60 \%)$ in the area and some unsuitable nests (remote location, nest already containing nestlings when discovered, etc.), data on nestling development and behavior could only be acquired from 25 nests. The tailorbird nests found in this study had clutches with 3 eggs $(n=2), 4$ eggs $(n=13)$ or 5 eggs $(n=11)$. After experimental manipulation, 13 of the nests contained one cuckoo nestling and 12 nests contained one host nestling. Nestlings fledged from 14 of the 25 nests, including 5 fledged cuckoos and 9 fledged tailorbirds. Nestlings had not yet fledged in 6 nests when the study ended, and the remaining 5 nests were predated during the study period.

\section{Morphology}

Development of characteristic traits of cuckoo nestlings is described in detail in Table 1, and photos visualizing the development of both tailorbird and cuckoo are presented in Fig. 2. The Plaintive Cuckoo, being altricial, hatches naked and blind after 13.0 days $(\mathrm{SD} \pm 0.5, n=5)$ of incubation, earlier than tailorbird nestlings, which hatch after 13.7 days ( $\mathrm{SD} \pm 0.7, n=11$ ) (Wang unpublished data). At hatching, the cuckoos had the same skincolor as host nestlings: pinkish with a pale yellow color on extremities, but the cuckoos were marginally larger and heavier [cuckoo hatchlings: $1.15 \pm 0.06, n=8$; tailorbird hatchlings: $0.79 \pm 0.06, n=6$ (Weight \pm SD in g)] and had a bit more robust body shape with a concave rump. Cuckoo nestlings gained weight faster than tailorbird nestlings (Fig. 3), and the growth patterns followed a sigmoidal growth curve.

In contrast to the weight trends, the tarsus grew more slowly in cuckoo nestlings compared to tailorbird nestlings and was almost the same in fledglings of the two species (Fig. 4). The gape color of cuckoo nestlings was red without any markings, turning deeper red as the cuckoo aged. By contrast, tailorbird nestlings had a yellow gape with two black tongue markings. The cuckoo bill was pale yellow and darkened with age, with only the tip remaining yellow at fledging. The pinkish skin color started to darken after 3 days of age, an effect emphasized by the emergence of teleoptiles pin feathers (all unsheathed flight and contour feathers). The eyes started opening around day 6 .

Cuckoo nestlings fledged just after 18 days of age $(n=5)$ when they had gained on average $24.1( \pm$ SD 0.91$)$ $\mathrm{g}$, and the fledglings possessed a juvenile plumage similar to the female Plaintive Cuckoo hepatic morph. Three nestlings fledged between day 18 and 19, one nestling fledged between day 19 and 20 and one nestling between day 19 and 21. Tailorbirds experienced faster development and hence a shorter nestling period than cuckoos. Feathers appeared and started unsheathing at an earlier point yet the eyes opened at around the same time as for cuckoo nestlings. Tailorbird nestlings fledged between days 12 and $13(n=4)$ when they reached a mean weight of 7.04 ( \pm SD 0.07). However, two individuals fledged at day 15 after abnormally slow development in one and louse infestation in the other.

\section{Eviction}

Newly hatched cuckoos were translocated to nests at different brooding stages, depending on nest availability. Hence most cuckoos experienced the presence of tailorbird nestlings that were always evicted $(n=9)$, including one individual that evicted a sole host nestling but

(See figure on next page.)

Fig. 2 Cuckoo nestling (left panel) and tailorbird nestling (right panel) developmental stages documented at 3-day intervals, from hatching on day 1 to just before fledging on day 18 (cuckoo) and day 12 (tailorbird) 


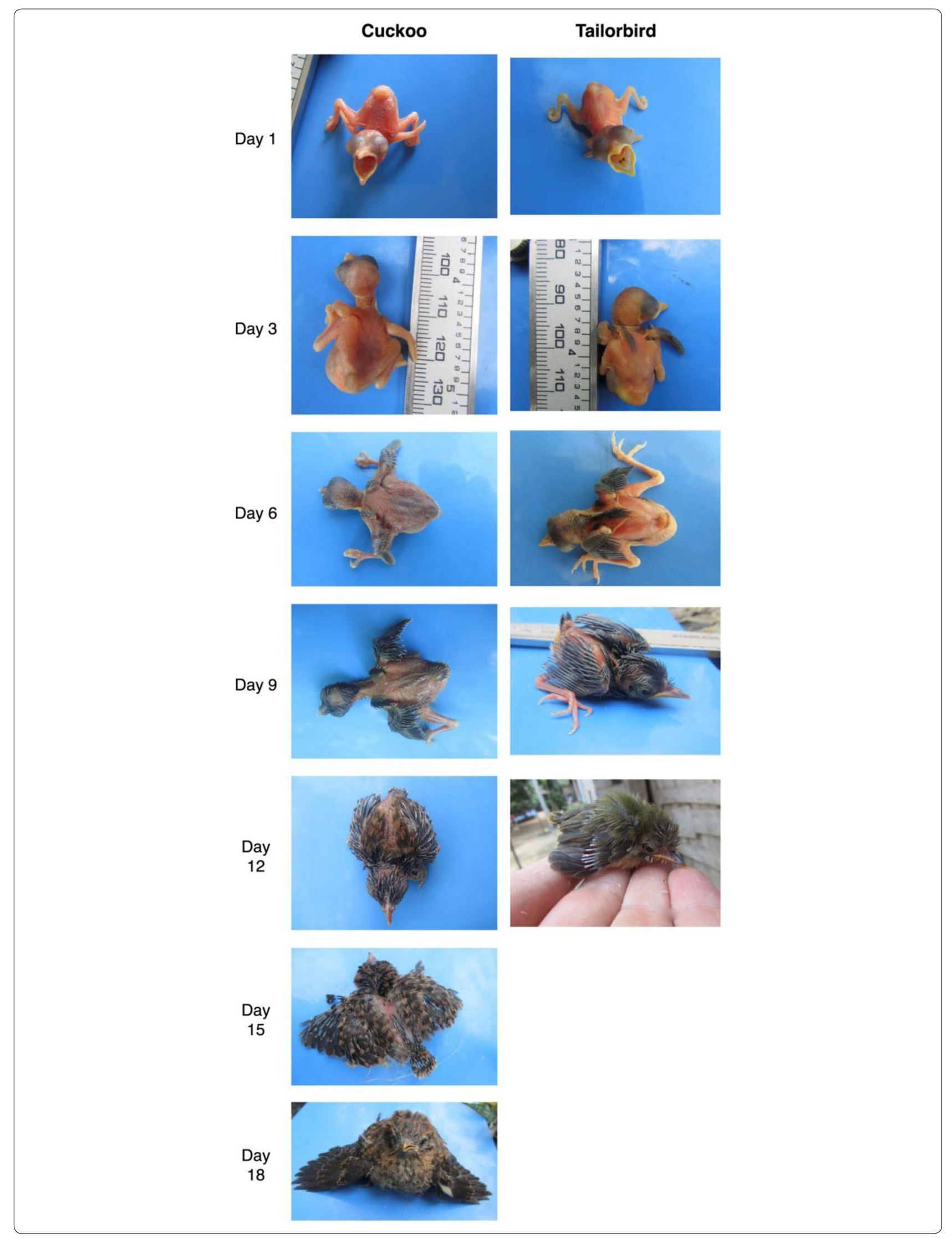


Table 1 Cuckoo nestling development characteristics, recorded at 3-day intervals

\begin{tabular}{|c|c|}
\hline Age (days) & Indicator characteristics \\
\hline $1-3$ & $\begin{array}{l}\text { Hatched with pinkish skin, yellow extremities, and grey eyelids. No neossoptiles (natal down feather) present. No pins were visible. A } \\
\text { prominent cavity on the lower back aided eviction efforts. The gape color was diffuse red/orange without any conspicuous markings. } \\
\text { The bill was pale yellow and the rictal flanges were a more saturated yellow color. This appearance lasted to day } 3, \text { when alar pins } \\
\text { started emerging in the subcutaneous layer and skin darkened }(n=12)\end{array}$ \\
\hline $4-6$ & $\begin{array}{l}\text { Skin darkened, appearing more red/purple/brown, with darker coloration over emerging teleoptiles. Remiges emerged and pierced the } \\
\text { skin on the alar tract. Rectrices on the caudal tract emerged and pierced the skin. The contour feathers emerged and pierced the skin } \\
\text { on the capital and ventral tracts. Pin feathers on the femoral and crural tracts appeared. Eyes started opening (earliest on day } 5 \text { ). The } \\
\text { base of the bill darkened, whereas the bill tip remained pale yellow and the rectal flanges brighter yellow. The gape turned deeper } \\
\text { reddish }(n=12)\end{array}$ \\
\hline 7-9 & $\begin{array}{l}\text { Pin feathers on the lower part of the dorsal tract emerged and together with the crural and femoral tract pierced the skin. All teleoptiles } \\
\text { showed rapid growth. The alar remiges pins turned paler at the outer end and started erupting. Eyes continued opening. The gape } \\
\text { turned deep red }(n=7)\end{array}$ \\
\hline $10-12$ & Ventral, femoral, capital, and caudal tracts erupted. Alar tract continued unsheathing. Eyes were open $(n=6)$ \\
\hline $13-15$ & $\begin{array}{l}\text { Dorsal tract started erupting. Extensive unsheathing occurred on all teleoptiles. Exposed feathers covered a large number of hidden pin } \\
\text { sheaths }(n=6)\end{array}$ \\
\hline $16-18$ & $\begin{array}{l}\text { The plumage appeared buffy and completed, mostly true for contour feathers. However, primaries and secondaries, as well as rectrices, } \\
\text { were unsheathed slightly more than halfway. Pin sheaths on dorsal tract were still visible }(n=5)\end{array}$ \\
\hline
\end{tabular}

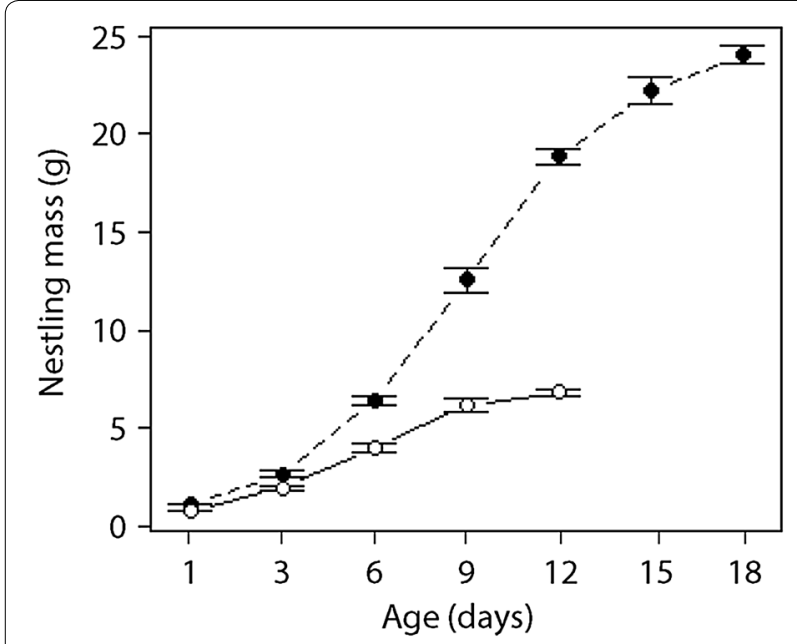

Fig. 3 Weight gain of cuckoo (filled circles) and host nestlings (open circles). Weight is shown in $\mathrm{g}$ on the $y$ axis and age is presented in days on the $x$ axis

none of the eggs. Cuckoo nestling eviction behavior was initiated at the earliest on day $2(n=4)$ and the behavior apparently ceased by day $5(n=3)$. Eviction was observed in 9 of the 13 nests. The three individuals where eviction was not observed had in common that all were reared in nests with eggs that never hatched and never experienced the presence of nestlings. One of them was observed to possess eviction behavior, but failed to evict the eggs over the nest rim. Another cuckoo nestling was found posthatching and estimated to be 6 days old, i.e. presumably after eviction behaviors were relaxed, but the parasite was found alone in the nest.

\section{Warning display}

Cuckoo nestlings developed an anti-predatory defense trait at 11 or 12 days of age whereby they, if approached by a potential threat, made themselves bigger by raising up in the nest, puffing out their feathers, lifting their heads, displaying their wings and red gape and striking intimidatingly at the threat. If the nestling was removed from the nest, it liberated sticky and smelling faeces. After this behaviour was acquired, not a single cuckoo nestling was predated $(n=6)$.

\section{Begging display}

Plaintive Cuckoo nestlings possessed exaggerated begging displays that were evident during the second half of the nestling period (after the warning display was attained). Whenever the host parents appeared in nest surroundings, nestlings shook their heads intensely, often while displaying their big, red gapes. Even after they had been fed, they continued their begging display. Parasite nestlings emitted strong begging calls that were similar in structure to those produced by tailorbird nestlings (Fig. 5). During the first days, the pitch of the call varied between the two species; the fundamental partial of cuckoo nestling calls was most frequently observed at $3-4 \mathrm{kHz}$, whereas the tailorbird calls were most often around 5-6 kHz. However, both species produced partials (several tones produced simultaneously) over a wide tone range. Between day 6 and day 9, the pitch of the partials often overlapped between the species calls. After day 9 , the begging calls of the two species started to diverge. Whereas the tailorbirds gave a drawn-out, noisy, or toneless "pccchhh" call, the cuckoo begging calls remained 


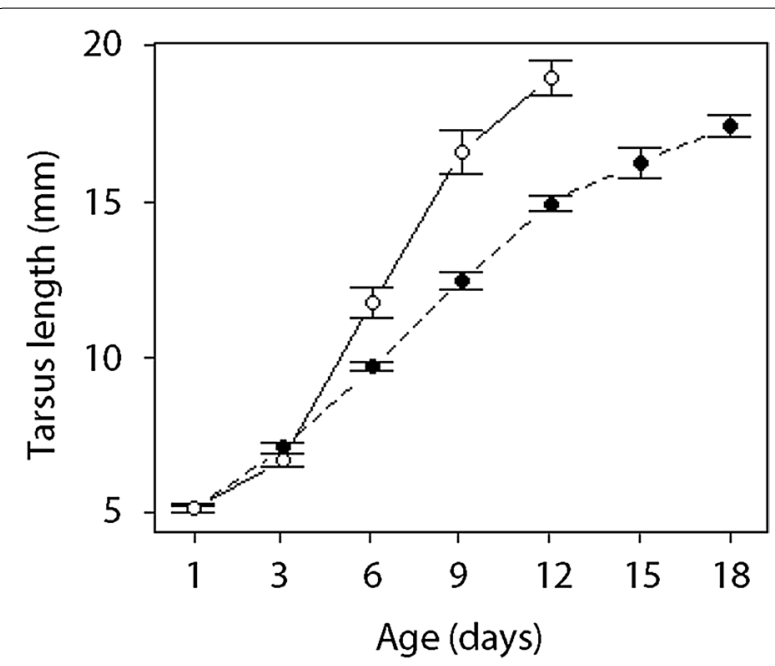

Fig. 4 Tarsus growth rate followed a sigmoidal growth curve with tarsus length in $\mathrm{mm}$ on the $y$ axis and age presented in days on the $x$ axis. The tarsus grew more slowly in cuckoo nestlings filled circles) than in tailorbird nestlings (open circles), and the maximum tarsus length is longer for tailorbirds than cuckoos

structured in partials of varying energy, but also here the partials became more blurred with increasing nestling age. Interestingly, the loudest partial changed from being the fundamental partial on day 3 and 6 to being the secondary or tertiary partial from day 9 in the cuckoo begging calls. Given the tonal complexity of cuckoo begging calls, which are drawn out during the latter period of the nestling stage, the sound output that host parents experience may not differ substantially from the noisier tailorbird nestling begging calls.

\section{Discussion}

Like other brood parasites that eliminate host progeny, Plaintive Cuckoo hatchlings were of larger mass and shaped differently than host progeny. During the first days post-hatching, the skin coloration was similar between the two species: pinkish skin, purple skin covering the eyes, and yellow beak. Morphological differences became evident with time. As tailorbird nestlings developed faster than parasites, emerging teleoptile pin feathers gave host nestlings a darker appearance. However, at the same time the skin coloration of cuckoo nestlings started to darken, eventually turning purple/black after 6 days of age.

Gape coloration of cuckoos was red and unmarked, in contrast to the tailorbirds' yellow gapes and black tongue marks. The emergent teleoptile plumage of the cuckoo was earth-brown and black; the upper part of the vane, the pennaceous, was brown, and the lower part, the plumulaceous, was black. This coloration was primarily evident in the contour feathers, but the flight feathers also showed it to some extent. The plumage of the juvenile cuckoo was similar to the adult plumage of the hepatic morph. As the tailorbird development was more rapid, it fledged after around 12 days in the nest. However, the cuckoo gained weight faster than tailorbird nestlings, and at fledging after around 18 days in the nest, cuckoos possessed about the same mass as 3.5 fledgeready tailorbird nestlings. This weight-ratio difference is less extreme than that observed for the Common Cuckoo nestlings raised by Reed Warblers; the cuckoos in that case were five to six times larger than their host counterparts when they fledged around day 17 . The actual fledging event coincided with the average fledging time of Common Cuckoos, which fledged between day 18 and 19 (Kleven et al. 1999).

Eliminating host progeny may be costly for cuckoos in terms of energy expenditure, and the annihilation of the host brood may reduce the signaling capability of cuckoo nestlings. Energy lost during eviction events may be regained, although it requires more time to do so; nestlings that evicted their competitors fledged with virtually the same mass as nestlings that did not perform evictions, but evictors fledged on average 1 day later (Grim et al. 2009). Plaintive Cuckoo nestlings exerted more efforts to evict host nestlings than host eggs; some individuals seemed unable to successfully evicting eggs or failed to recognize the presence of eggs altogether. Plaintive Cuckoo nestlings initiated eviction behavior on the second day post-hatching, while Common Cuckoo nestlings evicted the first offspring just after $40 \mathrm{~h}$ post-hatching; however, Common Cuckoo nestlings evicted eggs in 95\% of the eviction events (Honza et al. 2007).

Hypothetically, cuckoo nestlings should favor eviction of eggs over nestlings, as it appears to be less costly and the earlier host progeny are eliminated the better for the parasite. We observed the opposite pattern with most evictions happening at the nestling stage. Plausible mechanisms explaining this observation may relate to physiological constraints and ecological conditions. For example, there may be developmental constraints on an early initiation of eviction behavior as the earliest noted evictions were found at 2 days of age (i.e. nestlings need to exceed a developmental threshold before they can bear the costs of eviction). The effect of internal incubation may also be a less pronounced, as Plaintive Cuckoos hatch around $17 \mathrm{~h}$ before host hatchlings (Wang pers. comm.), while Common Cuckoo nestlings hatch $31 \mathrm{~h}$ earlier than their respective host progeny (Birkhead et al. 2010). In addition, nestlings may be hampered by deep and variable nest-shapes (Kleven et al. 1999; Grim 2006). 


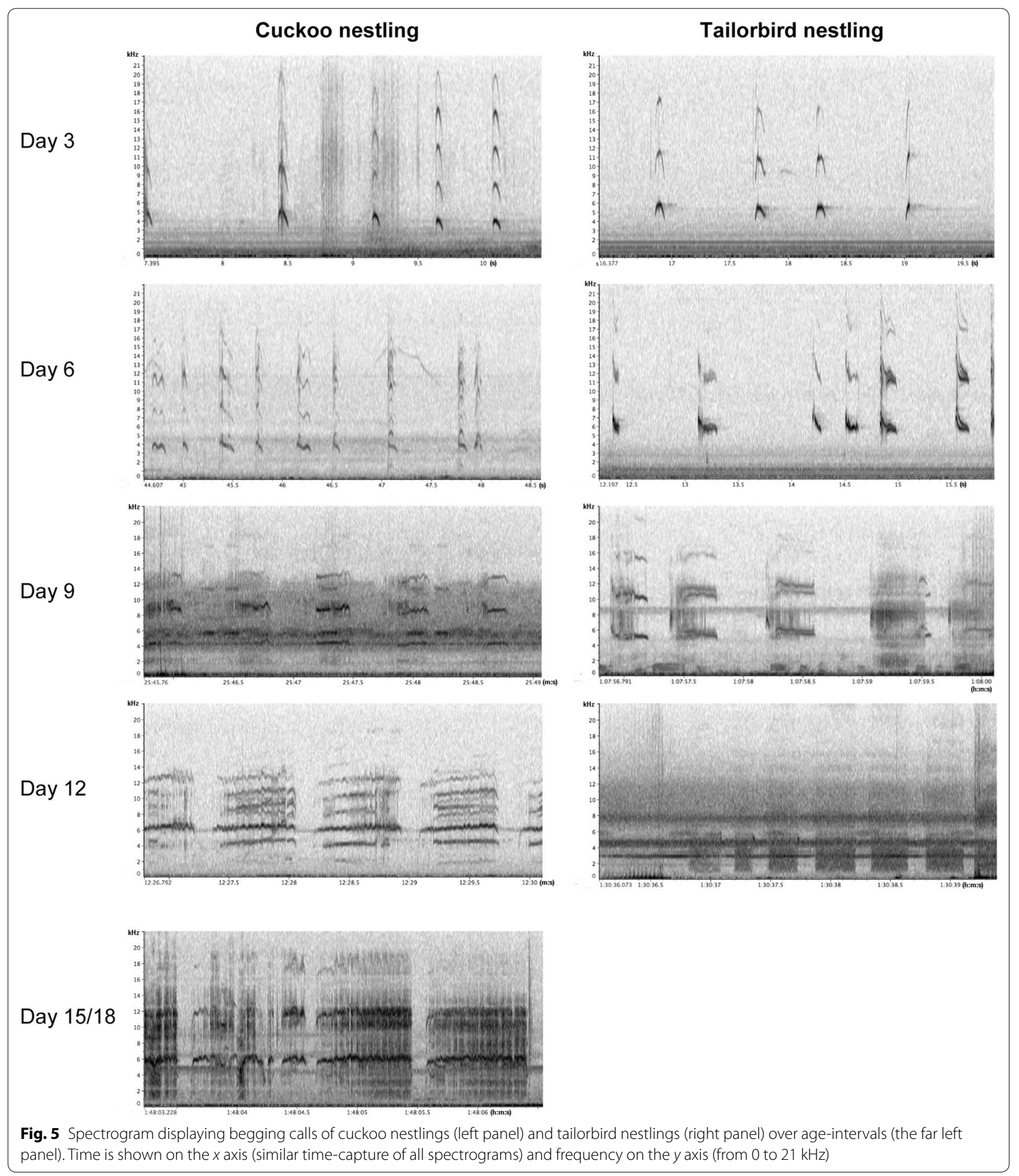

During the first phase of the nestling period, the begging display of cuckoo nestlings is rather reticent; nestlings sense the presence of host parents by sound or movement of the nest, to which they respond with headshaking, open gapes, and sometimes a high frequent sound (evident in recordings of food-deprived nestlings and occasionally observed in natural recordings). The behavior coincides with that of young tailorbirds, and 
both species increase the begging display after reaching the developmental stage of opened eyes. The experience of high predation rates during the first week in the nest also constitutes a potent selection force for cuckoo nestlings being cryptic during the first period of development. The visual stimuli intensified with age, prompting the increasingly larger cuckoo nestlings to puff their feathers up, display their red gapes, and shake their heads whenever parents were in the nest vicinity. When cuckoo nestlings were almost ready to fledge, they added occasional wing-lifting to the display, a relatively common behavior in fledglings (Grim 2008). Achievement of the warning display that is believed to combat predation could facilitate the development of the elaborated begging display. At fledging, cuckoo nestlings weighed around three and a half times that of fledging tailorbird nestlings-which represents just under one reproductive event, as tailorbirds most often laid four or five eggs in each clutch. However, cuckoos remained nestlings for longer than did their hosts and received care over a longer time scale than did host progeny.

Vocally, the sound output of one cuckoo nestlings seemingly equals the sound output of single tailorbird nestling up to day 9, after which begging call structure followed different trajectories with the tailorbird nestlings producing toneless calls and the cuckoos preserving the thrilling partials on distinct tones. This complexity in the tones of the cuckoo begging call becomes more obscure and noisy over time.

\section{Conclusions}

The developmental trajectory that we found for the Plaintive Cuckoo in this study is very similar to that of the well-studied Common Cuckoo. Both parasites share the selection towards host species that are of smaller size than themselves, and eliminate host offspring to acquire full parental care. Our findings will serve as a foundation for future work on the co-evolutionary interactions between the Plaintive Cuckoo and the Common Tailorbird, and hence constitute a first step towards understanding the plasticity of the Plaintive Cuckoo's exploitation of its 11 known hosts.

\section{Authors' contributions}

$\mathrm{OHT}, \mathrm{BGS}, \mathrm{WL}, \mathrm{ER}$ and FF designed the project. OHT and LW performed the field work with some assistance from the other authors including $\mathrm{CY}$ and AJ. OHT drafted the manuscript. All authors read and approved the final manuscript.

\footnotetext{
Author details

${ }^{1}$ Department of Biology, Norwegian University of Science and Technology (NTNU), 7491 Trondheim, Norway. ${ }^{2}$ Norwegian Institute for Nature Research (NINA), 7485 Trondheim, Norway. ${ }^{3}$ State Forestry Administration of China Key Laboratory for Biodiversity Conservation in Mountainous Areas of Southwest Karst, School of Life Sciences, Guizhou Normal University, Guiyang 550001,
}

China. ${ }^{4}$ Ministry of Education Key Laboratory for Ecology of Tropical Islands, College of Life Sciences, Hainan Normal University, Haikou 571158, China. ${ }^{5}$ College of Forestry, Guangxi University, Nanning 530004, China.

\section{Acknowledgements}

We would like to thank Qiuli Huang, Yungao Hu and Jianchou Nong for their assistance with fieldwork.

\section{Competing interests}

The authors declare that they have no competing interests.

\section{Availability of data and materials}

The datasets used and/or analyzed during the current study are available from the corresponding author on reasonable request.

\section{Consent for publication}

Not applicable.

\section{Ethical approval and consent to participate}

The experiments executed in this study comply with the current laws of China, where they were performed. Fieldwork was carried out under the permission of Nonggang National Nature Reserves, China. Experimental procedures were in agreement with the Animal Research Ethics Committee of Hainan Provincial Education Centre for Ecology and Environment, Hainan Normal University (HNECEE-2012-002).

\section{Funding}

This work was supported by the National Natural Science Foundation of China (31472013 and 31772453 to WL, 31672303 to CY, 31460567 to AJ) and The Research Council of Norway (218144 to BGS and FF).

Received: 19 June 2018 Accepted: 21 January 2019

Published online: 07 February 2019

\section{References}

Ackerman JT, Eagles-Smith CA. Accuracy of egg flotation throughout incubation to determine embryo age and incubation day in waterbird nests. Condor. 2010;112:438-46.

Álvarez F. The conspicuous gape of the nestling common cuckoo Cuculus canorus as a supernormal stimulus for rufous bush chat Cercotrichas galactotes hosts. Ardea. 2004;92:63-8.

Bioacoustics Research Program. Raven Pro: interactive sound analysis software (version 1.5). Ithaca: The Cornell Lab of Ornithology; 2014.

Birkhead T, Hemmings N, Spottiswoode C, Mikulica O, Moskát C, Bán M, Schulze-Hagen K. Internal incubation and early hatching in brood parasitic birds. Proc R Soc B. 2010;278:1019-24.

Britton N, Planqué R, Franks N. Evolution of defence portfolios in exploiter-victim systems. Bull Math Biol. 2007;69:957-88.

Davies NB. Cuckoos, cowbirds and other cheats. London: T \& AD Poyser Ltd.; 2000.

Davies NB, Kilner RM, Noble DG. Nestling cuckoos, Cuculus canorus, exploit hosts with begging calls that mimic a brood. Proc R Soc B. 1998:265:673-8.

Grim T. Low virulence of brood parasitic chicks: adaptation or constraint? Ornithol Sci. 2006:5:237-42.

Grim T. Experimental evidence for chick discrimination without recognition in a brood parasite host. Proc R Soc B. 2007:274:373-81.

Grim T. Begging behavior of fledgling rusty-breasted cuckoo (Cacomantis sepulcralis). Wilson J Ornithol. 2008;120:887-90.

Grim T, Kleven O, Mikulica O. Nestling discrimination without recognition: a possible defence mechanism for hosts towards cuckoo parasitism? Proc $R$ Soc B. 2003:270:573-5.

Grim T, Rutila J, Cassey P, Hauber ME. The cost of virulence: an experimental study of egg eviction by brood parasitic chicks. Behav Ecol. 2009;20:1138-46.

Honza M, Voslajerova K, Moskat C. Eviction behaviour of the common cuckoo Cuculus canorus chicks. J Avian Biol. 2007;38:385-9. 
Jonsomjit D, Jones SL, Gardali T, Geupel GR, Gouse PJ. A guide to nestling development and aging in altricial passerines. Washington, D.C.: U.S. Department of Interior, Fish and Wildlife Service Biological Technical Publication FWS/BTP-R6008-2007; 2007.

Kilner RM, Davies NB. How selfish is a cuckoo chick? Anim Behav. 1999;58:797-808.

Kilner RM, Noble DG, Davies NB. Signals of need in parent-offspring communication and their exploitation by the common cuckoo. Nature. 1999;397:667-72.

Kilner RM, Madden JR, Hauber ME. Brood parasitic Cowbird nestlings use host young to procure resources. Science. 2004:305:877-9.

Kleven O, Moksnes A, Røskaft E, Honza M. Host species affects the growth rate of cuckoo (Cuculus canorus) chicks. Behav Ecol Sociobiol. 1999:47:41-6.

Langmore NE, Hunt S, Kilner RM. Escalation of a coevolutionary arms race through host rejection of brood parasitic young. Nature. 2003;422:157-60.

Liang W, Yang C, Takasu F. How can distinct egg polymorphism be maintained in the rufescent prinia (Prinia rufescens)-plaintive cuckoo (Cacomantis merulinus) interaction—a modeling approach. Ecol Evol. 2017;7:5613-20.

Morales M, Murdoch D. Sciplot: scientific graphing functions for factorial designs. 2012. https://CRAN.R-project.org/package=sciplot. Accessed 20 June 2018.

Noble DG, Davies NB, Hartley IR, McRae SB. The red gape of the nestling cuckoo (Cuculus canorus) is not a supernormal stimulus for three common hosts. Behaviour. 1999;136:759-77.

Payne RB. The cuckoos. New York: Oxford University Press; 2005.
R Core Team. R: a language and environment for statistical computing, v3.4.4. R Foundation for statistical computing, Vienna. 2013. http://www.R-proje ct.org/. Accessed 15 May 2018.

Soler M, Martinez JG, Soler JJ, Møller AP. Preferential allocation of food by magpies Pica pica to great spotted cuckoo Clamator glandarius chicks. Behav Ecol Sociobiol. 1995;37:7-13.

Sorenson MD, Payne RB. A molecular genetic analysis of cuckoo phylogeny. In: Payne RB, editor. Bird families of the world: cuckoos. Oxford: Oxford University Press; 2005. p. 68-94.

Stevens M. Evolutionary ecology: knowing how to hide your eggs. Curr Biol. 2013:23:R106-8.

Svensson L. Identification guide to European passerines. 4th edn. Södertälje: British Trust for Ornithology; 1992.

Tanaka KD, Ueda K. Horsfield's hawk-cuckoo nestlings simulate multiple gapes for begging. Science. 2005;308:653.

Yang C, Huang Q, Wang L, Jiang A, Stokke BG, Fossøy F, Tunheim OH, Røskaft E, Liang W, Møller AP. Plaintive cuckoos do not select tailorbird hosts that match their own egg phenotypes. Behav Ecol. 2016;27:835-41.

Yang C, Liang W, Antonov A, Cai Y, Stokke B, Fossøy F, Moksnes A, Røskaft E. Diversity of parasitic cuckoos and their hosts in China. Chin Birds. 2012;3:9-32.
Ready to submit your research? Choose BMC and benefit from:

- fast, convenient online submission

- thorough peer review by experienced researchers in your field

- rapid publication on acceptance

- support for research data, including large and complex data types

- gold Open Access which fosters wider collaboration and increased citations

- maximum visibility for your research: over 100M website views per year

At BMC, research is always in progress.

Learn more biomedcentral.com/submissions 